\title{
Electromagnetic compatibility: new trends for new standards
}

\author{
Najett Neji and Raul de Lacerda \\ SUPELEC - DRE \\ 3 , rue Joliot Curie \\ 91192 Gif-sur-Yvette, France \\ Email: \{surname.name\}@supelec.fr
}

\author{
Felipe Nyland \\ UFSC - GEMCO \\ Caixa Postal 5024 \\ CEP 88040-970, Brazil \\ Email: nyland@gmail.com
}

\author{
Lionel Pichon \\ SUPELEC - LGEP \\ 11, rue Joliot Curie \\ 91192 Gif-sur-Yvette, France \\ Email: lionel.pichon@supelec.fr
}

\begin{abstract}
Interference between radio systems may occur if they share the same environment and use close frequencies at the same time. To ensure their successful coexistence, the Electromagnetic Compatibility (EMC) established rules and regulations within standardisation bodies and aims to protect these systems from harmful interference. Nowadays, with the proliferation of wireless communications and digital technologies, in addition to electronics evolution inside radio terminals, some additional parameters and new techniques are needed to represent more accurately the interference environment and guarantee radio systems coexistence. In this work, we provide an overview of the main standards related to EMC approaches and measurement techniques currently in use, and we show the benefits of considering time-domain parameters in the EMC analysis.
\end{abstract}

\section{INTRODUCTION}

The Electromagnetic Compatibility (EMC) determines necessary conditions to protect systems against harmful interference and undesired radiations. Systems and equipments should operate correctly in their environment and they must not cause harmful interference on other equipments.

To evaluate EMC, current standards are mainly focused on frequency-domain approaches that deal with worst-case situations where interference is assumed to be permanent. However, due to the advancement of technologies which includes the size of electronic components, wireless communication technologies are nowadays widespread around the world. As a consequence, radio coexistence is becoming hard to guarantee considering that spectrum is a limited resource. For this reason, it is necessary to evolve the standards to enable higher data rates, better spectral efficiency and intelligent frequency usage.

Hence, we emphasize in this paper that considering additional parameters in the theoretical studies'framework and employing other measurement techniques could improve EMC results. We propose to include these new aspects into current EMC standards.

The paper is organized as follows. First, in section II, we describe the state-of-the-art in EMC analysis approaches and measurement techniques. Then, in section III, we emphasize the influence of time-domain parameters on the EMC through a simple study case (some calculus have been developed in our previous work [1]). Finally, in section IV, we summarize the conclusions of the study.

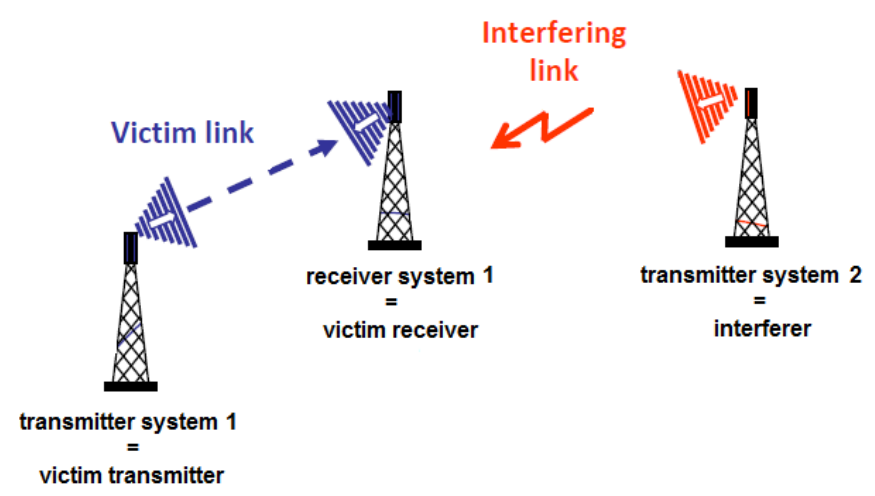

Fig. 1. EMC System model: transmitter, interferer and victim.

\section{STATE-OF-THE-ART: EXISTING EMC STANDARDS FOR TELECOMMUNICATION SYSTEMS}

Regarding telecommunications and information systems, there are standards in charge of establishing guidelines to keep equipment working within the EMC requirements. This means that they can coexist with other systems, without causing any interference or degradation in their functioning or causing losses in the quality of transmitted signals.

Besides the fast progress in technologies and electronic devices, the number of messages that need to be transferred is increasingly occupying the spectrum of frequencies. The various ways of conveying these signals (Wi-Fi, WiMAX, Bluetooth, LTE, etc.) compete for limited spectrum. This limitation led to a search for effective solutions that allow them to occupy a lower frequency bandwidth to transmit the same amount of information meeting the necessary conditions for their functioning.

To study the EMC, we represent two radio systems, system 1 and system 2, operating in the same environment. Interference between these systems may occur if they transmit at the same time using overlapping frequency bands. We illustrate in Fig. 1 the EMC scenario where the victim receiver (receiver of system 1) gets an interfering signal from the interferer (transmitter of system 2). 


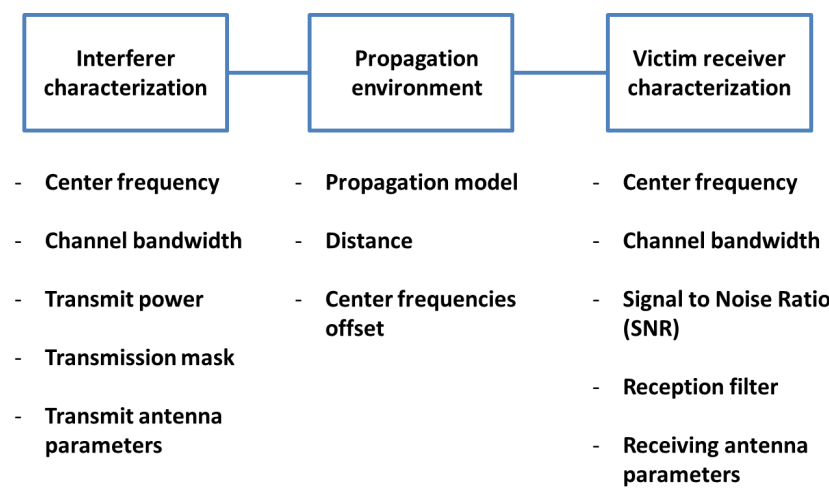

Fig. 2. Parameters for EMC study.

If the interference level $I$ is lower than the thermal noise level $N$ (both computed at the victim receiver input), the compatibility is guaranteed. For all the other cases, to ensure successful coexistence, current EMC standards usually specify a frequency and/or spatial separation in order to respect the required receiver performance for given signal level and quality of service.

This approach, called herein frequency-domain analysis, is based on five successive steps:

- Interference scenario identification,

- Interferer characterization,

- Victim receiver characterization,

- Interference path and victim path parameters determination,

- Link budget calculation.

We summarize in Fig. 2 the main parameters that should be considered in the EMC study.

The International Telecommunications Union, more particularly its Radio-communications sector (ITU-R), specify these parameters. To protect other systems operating in adjacent frequency bands, [2] and [3] provide spectral density limitations for out-of-band and spurious radiations, respectively. In addition, [4] specifies receivers selectivity and [5] their sensitivity and noise level. Antenna radiation patterns and maximal gains in certain frequency bands are also provided in the ITU recommandation 1336. Moreover, some ITU-R recommendations propose different propagation models to be used according to the interference scenario and frequency range, for example [6].

The ITU-R applies frequency-domain analysis with the previously mentioned parameters, to spectrum sharing studies (also named coordination studies) between radio systems (e.g. [7]). This methodology was also used in spectrum sharing studies within some European standardization bodies such as the Electronic Communications Committee (ECC) and the Conférence Européenne des administrations des Postes et Télécommunications (CEPT) (see [8] for example).

Most of these studies are based on Monte-Carlo simulations, using the (Spectrum Engineering Advanced Monte-Carlo Tool (SEAMCAT)) [9] software. The interference is evaluated in terms of probability that the Signal to Interference plus Noise Ratio (SINR) becomes lower than a specified minimum threshold by the user, and based on input physical layer parameters.

In conventional EMC testing, the interfering field strength at the victim receiver is required to be less than the regulatory levels recommended by the ITU. Hence, the radio spectrum has been divided into distinct frequency bands, usually with exclusive access.

However, spectrum resources become scarce as digital systems are being deployed to provide various services requiring higher data rates. Consequently, the ITU has kept some bands for unlicensed use to allow the development of new technologies. Systems operating in these bands are foreseen to use simultaneously the same or nearby frequency bands (case of Industrial, Scientific, Medical (ISM) bands). The frequency allocation is also changing with the emergence of digital systems (case of white spaces in the broadcast television spectrum). The radio environment is becoming complex also because of electronics evolution within radio devices (converged wireless communication devices). In addition, decentralized wireless networks (also named ad-hoc networks) are being widely developed and use complex technologies. These networks are increasing and use cognitive radios (CR) which are smart radios able to adapt their technologies depending on the user demand, the traffic load and propagation conditions.

Under these complex environments, radio systems are likely to suffer from interference and this is critical for their coexistence. Consequently, the ITU also recommends to refine the compatibility criteria based on link budget considerations for systems allocated in adjacent or nearby bands [10] or at the same frequency [11]. One other proposed solution by the is to limit transmit power spectral densities of systems operating in the ISM bands. Interference mitigation techniques are also mentioned in the literature, such as frequency hopping, Listen Before Talk [12] and Dynamic Frequency Selection [13]. These techniques use additional time-domain parameters but they are applied to specific spectrum sharing studies.

In addition, we still have a set of standards that cover all the electronic systems that are not intentional emission sources, as example, Comité International Spécial des Perturbations Radioélectriques CISPR 11 (for industrial, scientific, and medical apparatus) [14] and CISPR 22 (for Information Technology (IT) equipment) [15]. These standards specify the tests to be performed, electromagnetic fields limits to be respected, and check if the equipment under these regulations are compatible. In addition, CISPR-16-1-1 [16] determines the allowed equipment that can be used to perform these measurements.

Many electronic components can be very vulnerable to radio frequency pollution due to repetitive digital signals that have harmonics reaching the $\mathrm{GHz}$ range. This non-intentional energy can be either radiated or conducted.

The CISPR standards establish that the issue of measuring the emitted field by an Equipment Under Test (EUT) should preferably be done under two conditions. At first, EUT and the measurement system are inserted into an Open Area Test Site 
(OATS), free from any external Electromagnetic Interference (EMI). At second, tests could be performed inside "semi" anechoic chambers. In both settings, a calibrated antenna located at 3 and at 10 meters away from EUT, is connected to an EMI Test Receiver. It measures the emission of electromagnetic fields throughout the established frequency range (between 9 $\mathrm{kHz}$ and $6 \mathrm{GHz}$ ).

These tests usually take a long time to be concluded, because they are performed completely in the frequency domain. However, many recent works have questioned the guidelines imposed by CISPR. Problems involving standardization [17], chamber validation techniques [18], laboratory correlation studies [19] and correlations between measurements at 3 and 10 meters [20] have been addressed. The correlations between measurements at 3 and 10 meters are based on Maxwell equations and assume three conditions: a point source, a free-space environment, and a far-field environment. However, these conditions are not always necessarily satisfied. First, the majority of tested electronic products in EMC laboratories are distributed sources (they have a width, a height, and a length). Moreover, most measurements are not made in the farfield environment. Since near-field effects depend on effective size of source, effective size of the observer and the distance between source and observer, the antennas coupling would be very complex to model, mainly in low frequencies.

Many studies have also suggested new enhancements to improve the results in accordance with reality. Projects of better EMI receivers covering larger frequency ranges [21] and joint time-frequency analysis [22] were recently presented.

As far as EMI susceptibility is concerned, the International Electrotechnical Commission IEC 61.000-4-3 Standard [23] gives specified field levels subjected to the type of used EUT. The susceptibility tests are performed by sweeping single continuous wave signals through the test-frequency range. However, signals in the real RF environment are rather wideband signals having random variations in amplitude, frequency, and timing (for example Orthogonal Frequency Division Multiplexing (OFDM) signals). Hence, the coupling of EM fields is complex and it is difficult to predict EUT performance only based on a single test result.

\section{TIME-FREQUENCY APPROACH FOR EMC CALCULATION}

Current EMC analysis in the frequency domain deals with worst case scenarios, where the interferer is assumed to transmit continuously and the victim receiver is likely to intercept signals continuously. In some cases where the interference is still high, one common solution is to move the interferer away from the victim receiver and/or to change their relative orientation.

As the radio environment is becoming complex, EMI compliance is hard to guarantee and additional parameters are needed to represent more accurately the propagation environment. Time-domain parameters are interesting because the information within digital systems is transmitted through pulsed signals.
In this section, we propose to study the influence of the channel-occupation-rate parameter ( percentage of time of effective transmission by a system) on the coexistence between radio systems sharing the same spectrum. Let us take again the example shown in Fig. 1 where system 1 is the victim system and system 2 is the interfering system.

We call the respectively bandwidths of victim and interfering systems by $B_{1}$ and $B_{2}$ and their center frequencies by $F_{1}$ and $F_{2}$. In addition, we denote the total power of victim and interference signals at the victim's receiver by $P_{1}$ and $P_{2}$. Moreover, $H_{1}(f)$ and $H_{2}(f)$ are respectively the reception filter of system 1 and the transmission mask of system 2 . We also define the receptively channel occupation rates of both signals by $R_{1}$ and $R_{2}$. Finally, we suppose that both signals are independent.

Let us first compute the SINR before taking into account the channel occupation rates. The general expression of the SINR is :

$$
S I N R=\frac{S}{N+I}=\frac{1}{\left(\frac{N}{S}\right)+\left(\frac{I}{S}\right)}=\frac{1}{\left(\frac{1}{S N R}\right)+\left(\frac{1}{S I R}\right)},
$$

being $S N R$ the ratio $\frac{S}{N}$ and $S I R$ the ratio $\frac{S}{I}$.

$S$ and $I$ are the powers received by the victim from the victim transmitter and the interferer, respectively, both computed in the victim system bandwidth:

$$
S=\int_{B_{1}} \frac{P_{1}}{B_{1}} \cdot H_{1}(f) \mathrm{d} f
$$

and

$$
I=\int_{-\infty}^{+\infty} \frac{P_{2}}{B_{2}} \cdot H_{1}(f) \cdot H_{2}(f) \mathrm{d} f .
$$

In addition, $N$ is the noise level in the victim system bandwidth, related to the Boltzmann constant $k$ and the temperature $T$ (in $K$ ) by :

$$
N=k \cdot T \cdot B_{1} .
$$

Consequently, we have:

$$
\begin{aligned}
& \operatorname{SINR}= \\
& \left.\qquad \frac{\int_{B_{1}} \frac{P_{1}}{B_{1}} \cdot H_{1}(f) \mathrm{d} f}{\left(\int_{-\infty}^{+\infty} \frac{P_{2}}{B_{2}} \cdot H_{1}(f) \cdot H_{2}(f) \mathrm{d} f\right)+\left(k T B_{1}\right)}\right]
\end{aligned}
$$

For the victim system performance evaluation, we use the famous theorem of Shannon [24] which provides the error-free capacity under gaussian noise interference. We assume from now on that interference plus noise could be modeled as a white gaussian noise.

Theorem 3.1: (Shannon-Hartley Theorem [24]) The errorfree capacity (bits/second) that can be transmitted over a additive white gaussian channel is

$$
D_{\max }=B \cdot \log _{2}(1+S N R) .
$$


In our case, we call $C$ (in bits/channel access) the capacity of the victim system in the presence of noise and interference. Three cases are possible:

- Capacity $C$ without taking into account the channel occupation rates;

- Capacity $C^{\prime}$ based on the average $\operatorname{SINR}(\widehat{\operatorname{SINR}})$;

- Average capacity $C^{\prime \prime}$ based on the instantaneous SINR $(\operatorname{SINR}(t))$.

In the first case, the capacity of the victim system is:

$$
C=R_{1} \cdot \log _{2}(1+\operatorname{SINR})
$$

which means that:

$$
\begin{aligned}
C= & R_{1} \log _{2} \\
& \left(1+\left[\frac{\int_{B_{1}} \frac{P_{1}}{B_{1}} H_{1}(f) \mathrm{d} f}{\int_{-\infty}^{+\infty} \frac{P_{2}}{B_{2}} H_{1}(f) H_{2}(f) \mathrm{d} f+k T B_{1}}\right]\right)
\end{aligned}
$$

In the second case, the capacity of the victim system is computed based on $\widehat{\operatorname{SINR}}$ (calculus are developed in [1]) and its final expression is:

$$
\begin{aligned}
C^{\prime}= & R_{1} \log _{2} \\
& \left(1+\left[\frac{R_{1} \int_{B_{1}} \frac{P_{1}}{B_{1}} \cdot H_{1}(f) \mathrm{d} f}{R_{2} \int_{-\infty}^{\infty} \frac{P_{2}}{B_{2}} H_{1}(f) H_{2}(f) \mathrm{d} f+R_{1} k T B_{1}}\right]\right)
\end{aligned}
$$

In the third case, the average capacity of the victim system in the presence of the interferer considering $\operatorname{SINR}(t)$ (also see [1]) is given by:

$$
\begin{aligned}
C^{\prime \prime}= & R_{1} \cdot\left(1-R_{2}\right) \cdot \log _{2}\left(1+\frac{\int_{B_{1}} \frac{P_{1}}{B_{1}} \cdot H_{1}(f) \mathrm{d} f}{k T B_{1}}\right) \\
& +R_{1} \cdot R_{2} \cdot \log _{2} \\
& \left(1+\left[\frac{\int_{B_{1}} \frac{P_{1}}{B_{1}} \cdot H_{1}(f) \mathrm{d} f}{\int_{-\infty}^{+\infty} \frac{P_{2}}{B_{2}} H_{1}(f) H_{2}(f) \mathrm{d} f+k T B_{1}}\right]\right),
\end{aligned}
$$

We now compute the three capacities $C, C^{\prime}$ and $C^{\prime \prime}$ with respect to the ratio of system powers $\frac{P_{1}}{P_{2}}$. To simplify the study, we assumed that both system masks $\left(H_{1}(f)\right.$ and $\left.H_{2}(f)\right)$ are ideal, i.e. that they are expressed by :

$$
H_{1}(f)=\left\{\begin{array}{cc}
1 & F_{1}-\frac{B_{1}}{2} \leq f \leq F_{1}+\frac{B_{1}}{2} \\
0 & \text { otherwise }
\end{array}\right.
$$

and

$$
H_{2}(f)=\left\{\begin{array}{cc}
1 & F_{2}-\frac{B_{2}}{2} \leq f \leq F_{2}+\frac{B_{2}}{2} \\
0 & \text { otherwise }
\end{array}\right.
$$

We also consider that system 1 and system 2 use overlapping frequency bands, which means that the frequency offset between them $\delta$ (given by $F_{2}-F_{1}$ ) verifies

$$
-\frac{B_{1}+B_{2}}{2} \leq \delta \leq \frac{B_{1}+B_{2}}{2} .
$$

We finally assume that $B_{1} \leq B_{2}$, which means that the interfering signal occupies a larger bandwidth than the victim system.

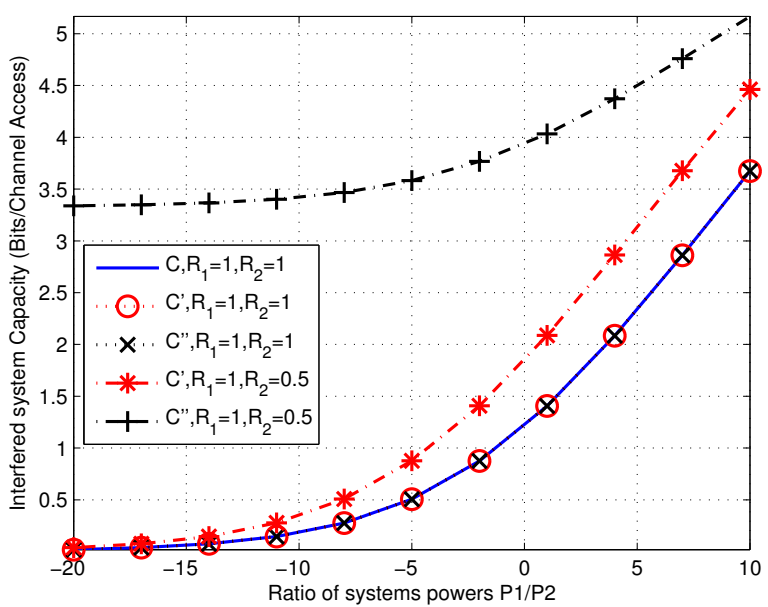

Fig. 3. : Channel capacity in the presence of co-channel interference and noise with respect to the ratio $\frac{P_{1}}{P_{2}}$, for $S N R=20 d B, \frac{B_{2}}{B_{1}}=2$ and $\delta=0$.

Under these conditions, the expressions of the three capacities are given by (calculus are developed in [1]):

$$
\begin{gathered}
C=R_{1} \log _{2}\left[1+\left(\frac{1}{S N R}+\frac{P_{2}}{P_{1}} \frac{B_{1}+B_{2}-2 \delta}{2 B_{2}}\right)^{-1}\right], \\
C^{\prime}=R_{1} \log _{2}\left[1+\left(\frac{1}{S N R}+\frac{R_{2}}{R_{1}} \frac{P_{2}}{P_{1}} \frac{B_{1}+B_{2}-2 \delta}{2 B_{2}}\right)^{-1}\right], \\
C^{\prime \prime}=R_{1}\left(1-R_{2}\right) \log _{2}(1+S N R)+R_{1} R_{2} \\
\log _{2}\left[1+\left(\frac{1}{S N R}+\frac{P_{2}}{P_{1}} \frac{B_{1}+B_{2}-2 \delta}{2 B_{2}}\right)^{-1}\right] .
\end{gathered}
$$

For the simulation results, we consider $\frac{P_{1}}{P_{2}}$ values between $-20 d B$ and $10 d B$ and we set the $S N R$ to $20 d B$. We investigate $R_{1}=1$ (continuous transmission by the victim) and two values of the channel occupation rate by the interferer: $R_{2}=1$ (continuous interference) and $R_{2}=0.5$ (half-time interference). Finally, for the results presented in this paper, we assume that $\frac{B_{2}}{B_{1}}=2$ and we take two values of systems center frequencies offset ( $\delta=0$ and $\delta=\frac{B_{1}+B_{2}}{4}$ ).

We notice from Fig. 3 and Fig. 4 that when both systems transmit continuously, the capacities $C, C^{\prime}$ and $C^{\prime \prime}$ are identical. When the average or instantaneous SINR is considered, the victim system capacity increases for both studied values of systems frequency offset. We also notice that the higher is the power ratio $\frac{P_{1}}{P_{2}}$, the higher is the capacity gain with average SINR, whereas the capacity gain with instantaneous $S I N R$ is the more significant for low values of $\frac{P_{1}}{P_{2}}$.

We can see in this simple example that the victim system performance increases significantly when channel occupation rates of both systems are considered. By taking into account the instantaneous SINR in the coexistence analysis, the result becomes more realistic. As a result, frequency sharing studies 


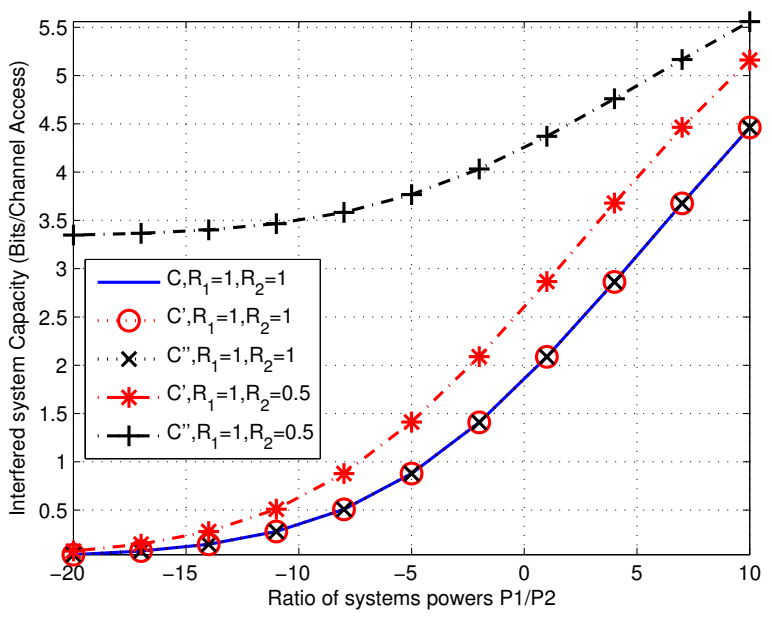

Fig. 4. : Channel capacity in the presence of co-channel interference and noise with respect to the ratio $\frac{P_{1}}{P_{2}}$, for $S N R=20 d B, \frac{B_{2}}{B_{1}}=2$ and $\delta=\frac{B_{1}+B_{2}}{4}$.

could be more precise and systems could be more accurately characterized.

We recall herein that we made approximations on the propagation environment to get simple SINR expressions using the channel occupation rates of both systems. We also studied the case where interfering transmission mask and victim reception filter are ideal and we considered that their antennas are isotropic. More results can be easily obtained using realistic masks/filters and antennas radiation patterns in our study case. To get more accurate results, additional parameters should be taken into account such as the Inter-Symbol Interference and multi-path phenomena.

\section{CONCLUSION}

In this paper, we summarized trends of technological advances and their consequences on EMC analysis. The difficulties and problems that go against current EMC standards were also discussed.

In addition, we showed the importance of time-domain considerations to improve the spectrum sharing between radio systems. Current EMC frequency domain analysis provides worstcase results (the involved systems are assumed to transmit continuously), whereas the time-frequency approach seems to be more accurate. It takes into account time-frequency aspects and technology properties. Through a simple study case, we emphasized the benefits of using time-domain parameters in the EMC study.

For instance, the growing technological advancement brings increasing challenges to the agencies in charge of designing EMC standards. It demands a increasingly faster response and more frequent updates to ensure that the electromagnetic compatibility is guaranteed. This work can be considered as an openess to new methods of measurements and simulations under more realistic conditions, according to these new technological realities.

\section{REFERENCES}

[1] N. Neji, R. de Lacerda, A. Azoulay and T. Letertre, "Electromagnetic compatibility from a time-frequency perspective," Revista Telecomunicaoes, vol. December, p. (accepted), 2011.

[2] International Telecommunication Union - Radiocommunication Sector, "Unwanted emissions in the out-of-band domain," Recommendation ITU-R SM.1541-1, 2002

[3] _ "Unwanted emissions in the spurious domain," Recommendation ITU-R SM. 329-10, 2007.

[4] - "Selectivity of receivers," Recommendation ITU-R SM 332-4, 1978.

[5] - "Noise and Sensitivity of receivers," Recommendation ITU-R SM 331-4, 1978.

[6] _ "Calculation of free space attenuation," Recommendation ITU-R P. 525-2, 1994.

[7] _ - "Frequency Sharing Between Systems in the Fixed-Satellite Service and Radio-Relay Systems in the Same Frequency Bands," Recommendation ITU-R SF. 355-4, 1992.

[8] Electronic Communications Committee (ECC), "Report from CEPT to the European Commission in response to task 3 of the Mandate to CEPT on the 900/1800 MHz bands - "Compatibility between LTE and WiMAX operating within the bands $880-915 \mathrm{MHz} / 925-960 \mathrm{MHz}$ and $1710-1785 \mathrm{MHz} / 1805-1880 \mathrm{MHz}(900 / 1800 \mathrm{MHz}$ bands) and systems operating in adjacent bands"," European Conference of Postal and Telecommunications Administrations (CEPT), Tech. Rep. 41 (Final Report), 2010

[9] ECO : European Communication Office, Spectrum Engineering Advanced Monte-Carlo Tool : SEAMCAT Handbook, January 2010.

[10] International Telecommunication Union - Radiocommunications Sector, "Compatibility analysis between a passive service and an active service allocated in adjacent and nearby bands," Recommendation ITU-R SM.1633, 1986.

[11] — , "An additional methodology for the evaluation of the effect of interference between radiocommunication networks operating in a shared frequency band," Recommendation ITU-R SM.1751, 2006.

[12] Qing Zhao, Wei Ren and Swami, A., "Spectrum opportunity detection: How good is listen-before-talk?" in Signals, Systems and Computers, 2007. ACSSC 2007. Conference Record of the Forty-First Asilomar Conference on, nov. 2007, pp. $767-771$.

[13] Electronic Communications Committee (ECC), "Use of the band 5725 $5875 \mathrm{MHz}$ for broadband fixed wireless access (BFWA)," European Conference of Postal and Telecommunications Administrations (CEPT), Tech. Rep., 2006.

[14] "Cispr11 ed. 5.1, industrial, scientific and medical equipment - radiofrequency disturbance characteristics - limits and methods of measurement," 2010.

[15] "Cispr22 ed. 6.0, information technology equipment - radio distrubance characteristics - limits and methods of measurement," 2008.

[16] "Cispr16-1-1 ed. 3.1, specification for radio disturbance and immunity measuring apparatus and methods - part 1-1: Radio disturbance and immunity measuring apparatus - measuring apparatus," 2010.

[17] M. Ianoz, "Standardisation problems related to conducted limits for power line communication equipment," vol. May, 2003, pp. 174-177.

[18] M.A.K. Wiles, "The impact of recent changes to cispr standards on emc anechoic chambers," vol. May, 2008, pp. 307-310.

[19] T.C Toh, "Electromagnetic interference laboratory correlation study and margin determination," IEEE Transactions on Electromagnetic Compatibility, vol. 51, pp. 204-209, 2009.

[20] H.F. Garn, E. Zink and R.Kremser, "Problems with radiated-emission testing at $3 \mathrm{~m}$ distance according to cispr 11 and cispr 22," vol. August, 1993, pp. 216-221.

[21] D. Festa and R. Grego, "A novel approach to the design of a fully cisprcompliant emc/emi receiver 10 hz âĂŞ 6 ghz," vol. November, 2008, pp. 297-301.

[22] W. Pan and D. Pommerenke, "Emi failure analysis techniques: Ii. joint time-frequency analysis," IEEE EMC Society Newsletter, vol. 226, pp. $31-34,2010$.

[23] "Iec 61.000-4-3 ed. 3.2,electromagnetic compatibility (emc) - part 43: Testing and measurement techniques - radiated, radio-frequency, electromagnetic field immunity test," 2010.

[24] C. E. Shannon, "Communication in the presence of noise," Proc. Institute of Radio Engineers, vol. 37 : pp 10-21.,1949. 